\title{
Applications of Nanotechnology in Cancer: A Literature Review of Imaging and Treatment
}

Niranjan Bhandare* and Ashwatha Narayana

Department of Radiation Oncology, Greenwich Hospital/Yale New haven Health system, Greenwich, CT, USA

*Corresponding author: Niranjan Bhandare Department of Radiation Oncology, Greenwich Hospital/Yale New haven Health system, Greenwich, CT, USA, Tel: 203-863-3743; Fax: 352-265-0759; E-mail: niranjan.bhandare@greenwichhospital.org

Received date: May 12, 2014, Accepted date: Oct 27, 2014, Publication date: Oct 31, 2014

Copyright: (c) 2014 Bhandare N, et al. This is an open-access article distributed under the terms of the Creative Commons Attribution License, which permits unrestricted use, distribution, and reproduction in any medium, provided the original author and source are credited.

\begin{abstract}
Recent advances in the application of nanotechnology in medicine, often referred to as nanomedicine, may revolutionize our approach to healthcare. Cancer nanotechnology is a relatively novel interdisciplinary area of comprehensive research that combines the basic sciences, like biology and chemistry, with engineering and medicine. Nanotechnology involves creating and utilizing the constructs of variable chemistry and architecture with dimensions at the nanoscale level comparable to those of biomolecules or biological vesicles in the human body. Operating with submolecular interactions, it offers the potential for unique and novel approaches with a broad spectrum of applications in cancer treatment including areas such as diagnostics, therapeutics, and prognostics. Nanotechnology also opens pathways to developing new and efficient therapeutic approaches to cancer treatment that can overcome numerous barriers posed by the human body compared to conventional approaches. Improvement in chemotherapeutic delivery through enhanced solubility and prolonged retention time has been the focus of research in nanomedicine. The submicroscopic size and flexibility of nanoparticles offer the promise of selective tumor access. Formulated from a variety of substances, nanoparticles are configured to transport myriad substances in a controlled and targeted fashion to malignant cells while minimizing the damage to normal cells. They are designed and developed to take advantage of the morphology and characteristics of a malignant tumor, such as leaky tumor vasculature, specific cell surface antigen expression, and rapid proliferation. Nanotechnology offers a revolutionary role in both diagnostics (imaging, immune-detection) and treatment (radiation therapy, chemotherapy, immunotherapy, thermotherapy, photodynamic therapy, and anti-angiogenesis). Moreover, nanoparticles may be designed to offer a multifunctional approach operating simultaneously as an effective and efficient anticancer drug as well as an imaging material to evaluate the efficacy of the drug for treatment follow-up. In recent years, nanomedicine has exhibited strong promise and progress in radically changing the approach to cancer detection and treatment.
\end{abstract}

Keywords: Nanotechnology; Cancer treatment; Imaging

\section{Introduction}

Carcinogenesis is associated with progressive modifications/ alterations in the cellular, genetic, and epigenetic characteristics that results in uncontrolled cell division, ultimately leading to the formation of a malignant mass. Cancer is identified by unregulated, uncontrolled tissue growth. Resulting malignant tumors (cancer) may invade other adjacent organs, and spread to other locations (metastasis) farther from the location of its initiation, making the disease life-threatening. Conventional cancer diagnoses and treatments are based on clinical and pathologic staging. Diagnostics tools used to determine the stage include radiology and histopathology examinations. A limitation of conventional cancer screening technologies includes insufficient sensitivity and specificity in detecting precancerous conditions or early-stage cancer with a low rate of false positives, inability to determine tumor stage, and high cost [1].

Despite decades of advances, commonly used cancer treatments like chemotherapy, radiation therapy, and hormone therapy remain suboptimal. Radiation therapy may cure various cancers if they are restricted to a specific part of the body, but it is associated with acute early and delayed morbidities as well as the possibility of second malignancy. The limitations of the current cancer therapeutics includes nonspecific dispersin of anticancer drugs, insufficient delivery of drug concentrations to the target site, unacceptable toxicity, limited ability to track and control the site-specific therapeutic responses and adverse effects, and the development of drug resistance [2-4]. Conventional diagnostic classifications and prognostic estimations are inadequate to predict the success of the treatment [5]. Recent advances in cancer research have expanded our understanding of cancer as a disease at the genetic, molecular, and cellular levels, opening venues for research for the development of newer approaches and technologies.

Nanotechnology involves a convergence of multiple areas of science, and includes not only medicine but also physics, chemistry, biochemistry, and molecular biology. It explores previously unexplored physical characteristics and attributes (optical, magnetic electronic, catalytic) [6,7]. Nanotechnology offers significant potential benefits over conventional drug construction and distribution that may alter pharmacokinetics and distribution, improving efficacy and reducing side effects [8]. The size and surface properties associated with nanoparticle surfaces enhances drug portability, mobility and detainment $[9,10]$, increases selective confinement in tumor tissue, and makes them potentially effective tumor delivery vectors [11]. Nanotechnology, with progression in building multiplex-nanoparticles comprising of two or more components, expands functional capabilities for concurrent imaging and drug delivery [12]. 
Nanoparticles are specified as solid colloidal particles varying in dimensions from $10 \mathrm{~nm}$ to $400 \mathrm{~nm}$. They consist of macromolecular substances in which the reactionary agent (biologically active material or a pharmacologically active drug) is encapsulated or dissolved, or entrapped, or to which the active agent is attached or adsorbed [13].

In order to develop a novel, revolutionary approach to cancer research, the National Cancer Institute initiated an alliance for nanotechnology in cancer [14].

\section{Nanoparticles in Drug Delivery and Imaging}

Nanoparticles offer efficient and versatile tools for cancer detection (imaging) and treatment (drug delivery).

\section{Lipid-based Nanoparticles and Polymers}

These nanoparticles based on either lipids or polymers are often flaccid. Their size varies from $30 \mathrm{~nm}$ to over $100 \mathrm{~nm}$. Due to their flaccidity and biochemical interactions with components of cellular membrane they can travel through the cell membrane into cytoplasm [15].

\section{Liposomes}

Liposomes are globular, closed-colloidal vesicles consisting of a lipid bilayer that often contains phosphatidylcholine-enriched phospholipids and may also include mixed lipid chains possessing surfactant properties such as egg phosphotidylethanolamine and other lipid components like cholesterol that surround a central aqueous space [5]. The major types of liposomes are the multilamellar vesicle, the small unilamellar and large unilamellar vesicles, and the cochleate vesicle. Owing to the diversity of their structures and compositions, liposomes have become versatile tools in clinical applications in cancer treatment (imaging and therapy) [5,16-19]. While antigen sequences of variable length, immunomodulatory components, and hydrophilic agents can be enclosed in the aqueous interior, hydrophobic agents can be associated with the bilayer making liposomes an efficient receptacle for conveyance [20,21]. Modifications in drug encapsulation have indicated improvement to work under different environments [22]. Incorporation of hydrophilic polymer polyethylene glycol (PEG) attached to the exterior of the membrane allows these liposomes (referred to as sterically stabilized or Stealth) to avert apprehension by the immune system, especially the reticuloendothelial system. Being inert to interactions with plasma proteins and mononuclear phagocytes, the PEG coating greatly prolongs the duration of circulation while the drug is distributed [5,23-25]. Examples of presently available liposomal delivery systems include doxorubicin and daunorubicin. Improved accumulation in tumor, increased antitumor activity, and diminution in cardio toxicity of doxorubicin was observed after its encapsulation with anionic liposomes [26,27]. In ovarian and breast cancer treatment, liposomal doxorubicin was observed to be clinically secure and potent $[5,28,29]$.

Flexibility of liposome construction allows incorporation of imaging agents into either the bilayer or interior, making them effective carriers for intensification of contrast in magnetic resonance imaging (MR) and computed tomography (CT) [30,31].

Though free synthetic peptides are known to cause a poor immunogenic response, the use of peptides with oligomanose-coated liposomes may cause a strong cellular reaction $[2,22,26]$. Furthermore, diminished encapsulation potency, fast drainage of water soluble pharmaceutical in the presence of blood ingredients, and diminished stableness when stored have restricted the advancement of liposomes.

\section{Solid lipid nanoparticles (SLNs) and nanostructured lipid carriers (NLC)}

The inadequacy of secure polymers approved by regulatory agencies and their cost have restricted the extent of their utilization in clinical applications [32]. Lipids are offered as substitute transporters for lipophilic agents to compensate for these limitations of polymeric nanoparticles. The need for an alternate approach based on lipid components other than phospholipids that may let enhanced control over drug transport and dissemination of therapeutic agents that don't potently incorporate in to liposomes has led to the development of solid lipid nanoparticles (SLNs) and nanostructured lipid carriers (NLC). The in vitro and in vivo stabilization of SLNs and NLCs are commonly achieved through PEGylation or polymer coating (e.g., PEG2000, polyvinyl alcohol, poloxamers) [3], which have already been used for doxorubicin-formulated liposome stabilization (i.e., Doxil).

SLNs are colloidal transporters developed as a substitute to other traditional carriers (emulsions, liposomes, polymeric nanoparticles) and manufactured from synthetic/natural polymers satisfactory to optimize drug transport and diminish toxicity. SLNs have emerged as a variable substitute to liposomes as drug carriers. SLNs are submicron-dimensional lipid emulsions where the liquid lipid is replaced with solid lipid. These lipid-based nanoparticles are progressing as an efficient nontoxic alternative to lipophilic colloidal drug carriers. Encapsulation of a drug solution into an SLN can be performed using numerous methods, including high-pressure homogenization, microemulsion formation, emulsification-solvent evaporation (precipitation), solvent injection (or solvent displacement), phase inversion, the multiple emulsion technique, ultrasonication, and the membrane contractor technique [3]; they are produced by high-pressure homogenization $[33,34]$ or microemulsion-based techniques [35]. SLNs can be manufactured to transport lipophilic or hydrophilic or lipophilic pharmaceuticals, thus offering an optimal particulate carrier system. The versatility is emphasized by their colloidal size and their regulated release that enable drug safety through delivery by parental and non-parental tracks $[15,36,37]$.

NLCs are created using a combination of solid and liquid phase lipids, similar to SLNs. Appropriate lipid selection is critical for creating a stable drug-associated NLC. The type of solid lipid incorporated into the NLC determines the chemical strength and security of the pharmaceutical. In addition, the incorporation of drug into lattice defects of the NLC may alter particle stability [3]. The percentage of liquid-phase lipids included can affect the dimension and surface morphology of particles and the acid concentration controls the initial rate of drug release [38]. Depending on the structure of their lipid matrix: the imperfect type, multiple types, and amorphous or structure-less type, NLCs may be subdivided into three classes. The imperfect type of NLC has the least amount of liquidphase lipid (oil) and is composed of saturated and unsaturated lipids with varying fatty acid chain lengths, which lead to defects in the lipid matrix and compartments for drug storage [39]. The imperfect NLC is prone to an expulsion of drugs during the crystallization process of production. The multiple type of NLC avoids this drug expulsion by incorporating a higher concentration of liquid-phase lipids in the lipid matrix. The amorphous type of NLC forms a solid lipid that lacks any 
crystalline structure. This is achieved through the use of lipids such as hydroxyoctacosanylhydroxystearate and isopropyl-myristate [40].

The advantages of therapeutic drug delivery by SLN and NLC include (i) controlled and extended drug release, (ii) encapsulation of various drugs, and (iii) extension of blood dissemination time and utilization of the increased permeability and retention effect for enhancing treatment $[3,9]$. Hydrophobic drugs with brief circulation half-lives are a suitable contender for delivery via SLN and NLC. Usefulness of SLNs as drug transporters to treat cancer is reported [9]. To protract dissemination of the pharmaceutical after intravenous delivery for breast cancer and to enhance the permeability and retention effect, tamoxifen has been incorporated into SLNs [41]. SLNs consigned with methotrexate [42] and camptothecin [43] have been used to achieve tumor targeting. To lower morbidity and enhance the security and bioavailability of mitoxantrone-loaded SLN, local injections have been used [44]. Incorporation in an SLN has been observed to enhance the efficacy of doxorubicin [45]. The anticancer therapeutics paclitaxel and doxorubicin loaded into NLCs have been found to overcome cell-multi-drug resistance [46]. Compounds with the deaza skeleton of the antitumor drug temozolomide have also shown promising anticancer results when loaded in NLCs [47].

\section{Biodegradable polymeric nanoparticles}

Biodegradable polymeric (BDP) nanoparticles are submicron-sized particles with drug molecules diffused, entangled, adsorbed, linked, or encapsulated into the NPs or a gene of interest encapsulated inside a polymeric matrix. Continued pharmaceutical dispensation at the destination lasting over periods of up to weeks is allowed by using biodegradable materials for NP preparation [48]. There are two conventional mechanisms for preparation of nanoparticles (i) by distributing polymers that are preformed and (ii) polymerizing monomers [49]. Because of the variation in their purity and the need to crosslink that may lead to denaturation of associated pharmaceuticals, naturally occurring polymers (i.e., proteins and polysaccharides) are not commonly utilized for this operation. Noted for their biocompatibility and resorbability by natural processes, poly (lactic acid) (PLA) or poly (glycolic acid) (PLGA) are commonly used as a substitutes, [50]. In spite of being the most widely used polymer, PGLA is not the best transporter for operations pertaining to all drug applications. Drugs are successfully dispensed in the brain by Polybutylcyanoacrylate nanoparticles. Because of their muco-adhesive characteristics and capability to contain various bio-active molecules, other cyanoacrylate-based nanoparticles like polyalkylcyanoacrylate (PACA) and ethylcyanoacrylate (PECA) are regarded as up-andcoming drug-dispensing systems [50-52]. The dispensing of anticancer pharmaceuticals using nanospheres comprised of biodegradable hydrophobicpoly(-caprolactone)(PCL) and hydrophilicmethoxy poly(ethylene glycol) (MePEG) was tested [53].

In view of their properties, such as easy to synthesize, relatively inexpensive, biocompatible, non-immunogenic, non-toxic, water soluble, BDP nanoparticles are of great interest as an effective method for dispensing pharmaceuticals to particular tissues/organs, as a means of DNA gene therapy, and in their capability to dispense proteins, peptides, and genes by oral administration $[54,55]$.

These polymers can be effectively used for the controlled dispersion of a number of agents like insulin, human growth hormone, vaccines, anti-tumor agents, and contraceptives. Cancer-related drugs such as paclitaxel, doxorubicin, 5-fluorouracil, 9-nitrocamptothecin, cisplatin, triptorelin, dexamethasone, and xanthone,among others, have been successfully associated with PLGA [56]. Nanospheres comprised of naturally degradable hydrophobicpoly(-caprolactone) (PCL) and hydrophilicmethoxy poly(ethylene glycol) (MePEG) have been tested for delivery of taxol [53].

\section{Polymeric Micelles}

A micelle is an aggregation of surfactant molecules with both hydrophilic and hydrophobic components that undergo self-assembly in an aqueous surrounding to form compositions with a hydrophobic center with a stable hydrophilic encapsulation. Incorporating a generation of monomer $(\mathrm{G})$ in the main core, they are assembled by divergent (outward from core) [57] or convergent (inward towards the core) [58] methods [58].

Micelles have offered great promise in therapeutics as colloidal carriers for hydrophobic pharmaceuticals for cancer treatment $[59,60]$. Characteristics that make polymer micelles attractive include their smaller size, making them suitable for percutaneous lymphatic transport or extravasation from blood vessels into target tissue by enhanced permeation and retention effect, capability of dissemination of significant quantities of relevant material (e.g., small molecules, proteins, DNA/RNA therapeutics), enhanced in vivo steric stability in comparison to alternative transporters like liposomes, and proper selection leads to avoidance of fast absorption by the reticuloendothelial system. This increases the circulation time in the body $[61,62]$.

A number of preclinical studies showed that targeting polymer micelles associated with low water solubility anticancer pharmaceuticals enhance the disseminated concentration of pharmaceuticals to target tissue and lower the morbidities and toxicities in animal models $[63,64]$. Their applications extend to both diagnosis and treatment of cancer. Their use has been explored in photodynamic therapy [65], boron neutron capture therapy [66], and hyperthermia utilizing gold nanoparticles [66]. Use of various inactively focused micelles compounds has been examined in clinical trials. One of these (Genexol-PM) has obtained FDA-approval for utilization in breast cancer patients [59]. At present, the focus in micelle-based cancer therapeutics is placed on versatile micelles offering at least two out of three functions as targeting ligands, imaging agents, or triggered release. The mixing of imaging aspects and triggered release allows the possibility to follow the path of micelle inside the body and trigger its dissemination after reaching the targeted tissue, which enhances the efficiency of drug dissemination [59]. Combining imaging and focused targeting follow the path of the micelles along its course in the body, and to determine its delivery and dissemination at a specific targeted location.. The mixing of active targeting and triggered release results in enhanced cytotoxicity and antitumor activity in comparison with use of micelles that are not as versatile.. Triggered release may also be included in combination with active targeting. The combination of all three features is yet to be investigated.

Incorporation of polymeric micelles in nuclear imaging, MRI, and $\mathrm{X}$-ray CT, significantly contributes to the diagnosis of cancer and evaluation of treatment response [59]. Utilization of micelles conjugated with gamma emitters (99mTc and 111In) have been thoroughly examined for analyses of non-invasive biodistribution $[59,67,68]$. The inclusion of iron oxide particles in micelles or the utilization of chelators for combination of paramagnetic metals to the hydrophilic block of micelle-forming block copolymers are the two methods utilized for developing micelle-based MRI contrast agents 
[59,69-72]. Multifunctional polymeric micelles with imaging contrast agent and clusters of magnetic nanoparticles for efficient MRI and chemotherapeutic agents targeting ligands have been developed $[73,74]$.

\section{Dendrimers}

Dendrimers are highly branched synthetic polymers that are symmetric at the center and form spherical 3-dimesional micromolecules [75]. They are presented with two kinds of structural variants: One has a central core with spherical organization that leads to a branching polymer. The three parts of these dendrinmer include: a core, an inner shell, and an outer shell. The center is comprised of ethylene diamine (EDA), diaminobutyl (DAB), polyamidoamine (PAMAM), and polypropylimine (PPI) with various exterior residues like amine, carboxyl, and alcoholic groups. The dimension of the dendrimer also referred to as the "generation" of the dendrimer increases with the increase in the amount of primary amine groups linked to the center [76]. The other does not have a central core and comprises only of a number of polymers that exhibit significant branching. Though the functional group on the molecular surface determines the properties in most cases, there are dendrimers with internal functionality [77-79]. In most ideal cases, a dendrimer can be manufactured with supplementary components suitable for a specific purpose to exhibit various functions in different parts and to manipulate characteristics like solubility and thermal stability. The dimension and amount of branching on the dendrimer may be accurately defined by the process of synthesis. By functionalizing their outer shell with charged species or other hydrophilic groups, dendrimers can be made water soluble, unlike most polymers. Divergent and convergent synthesis are two known processes for dendrimer synthesis. The steps needed to protect the active sites make the manufacturing difficult and expensive.

With alterable size and shape, the branched construction generates increasingly functional exterior surface suitable to conjugate ligand and to serve as an attractive modality for drug delivery and imaging $[58,80,81]$ while the branching creates a core highly suitable to entrap pharmaceuticals and imaging agents $[82,83]$. The confined dimensions, efficiency of construction, ability to chemically modify the surface to enhance functional capabilities by conjugation with a variety of ligands extends versatility to carry multiple functions including delivery of imaging agents and cytotoxic pharmaceuticals to targeted locations. The general skeletal structure of dendrimer-drug conjugation is comprised of a covalent bonding between an anticancer agent and a group exterior to dendrimer. This approach extends distinct benefits over encapsulation of anticancer agent. With this method, a number of anticancer agents, cytotoxic pharmaceuticals, and targeting entities like folic acid, monoclonal antibodies, and peptides can be conjugated with an individual dendrimer therapeutic molecule. The dispersion of the agent is partly controlled by the type of conjugation and process enhanced specificity of dispersion. Malignant cells exhibiting enhanced expression of folic acid receptors are selectively targeted by dendrimers conjugated with folic acid [84-86]. Poly(glycol) dendrimer complexes exhibit increased aqueous solubility [87]. Melanin-conjugated dendrimers were utilized to enhance solvability of aniticancer drugs such as methotrexate and 6mercaptopurine and to lower the toxicity of the agents [88]. The use of dendrimer in dispensing agents for photodynamic therapy were examined for understanding its inter-cellular differentiation and selectiveness, retention characteristics, and pharmacokinetics.. Utilization of a Poly (amidoamine), or PAMAM, dendrimer system for neutron capture therapy has been investigated [66,89-91]. Preparation of dendrimer gold nanopadrticles enclosed inside a dendrimer has been tested for its utilization in photothermal treatment of cancerous tumors [66,92]. Recently, dendrimers conjugated with Gadolinium (Gd) paramagnetic contrast agents for MRI have been tested to study possible improvements in selective targeting as well as contrast and clearance characteristics [66,84]. Use of iodinated contrast agents based on dendrimers encapsulating a PEG center of differing lengths for potential CT imaging of tumor microvasculature have been reported [93].

\section{Inorganic Nanoparticles for Therapy and Imaging}

Inorganic nanoparticles are smaller in dimension $(5-40 \mathrm{~nm})$ and constitute a distinctly separate group of nanoparticles. They lack the flexibility of liposomes or polymeric nanoparticles. In its basic form, inorganic nanoparticles are comprised of an inorganic center and bioadaptable encapsulation which offers steadiness under differing interbody environments [48]. After successful utilization for imaging and treatment of malignancies both in vivo and vitro inorganic nanoparticles show optimism for clinical trials for both imaging and treatment. A number of applications of inorganic nanoparticles have been developed in the last decade [15,94].

\section{Quantum Dots}

Quantom dots (QDs) are nanocrystals composed of semiconductor substance. QDs are sufficiently small (ranging from 2-10 $\mathrm{mm}$ in diameter) to demonstrate quantum mechanical properties. QDs range from 2-10 $\mathrm{nm}$ in diameter and are composed of an inorganic elemental core (eg. cadmium, mercury) encompassed by a metal shell. QDs are comprised of 10-50 atoms and held electron hole pairs to discrete quantized energy levels. QDs fluoresce by electron excitation when excited with ultraviolet light $[95,96]$ with intrinsic emission fluorescent spectra wavelengths between 400 to $2000 \mathrm{~nm}$ [95]. The frequency range of emitted light is determined by band gaps associated with its composition and are inversely proportional to their size [97].

Developed about 20 years ago for electronics and optics, the use of QDs in research and applications in biomedicine with a wide range of applications in cancer treatment and research has significantly grown in recent years $[98,99]$. QDs have exhibited superiority to organic dyes in several ways including their ability to allow discrete narrow frequencies, their brightness due to their high extinction coefficient along with comparable quantum yield to fluorescent dyes [100], and their stability (allowing significantly less photobleaching) [101]. When manufacturing QDs that can be excited by one light source but emit light of varying wavelength it is possible to label and identify a number of biological objects of clinical significance [102,103].

QDs offer a multipurpose nanoscale framework for defining and constructing versatile nanoparticles that can be utilized to carry out both functions, in imaging and treatment [2]. The improved photostability of QDs have made them fit to track the activities at molecular and cellular levels in real time over a significant duration of time, and such observations have been carried out in lymph nodes of mice over a period of 4 months [104] for sensitive cellular imaging by acquiring multiple successive focal-plane images of cellular components that can be used to construct 3-dimensional images with higher resolution [105]. It is possible to use antibodies [106], peptides [107], DNA [108], nucleic acid aptamers, or small molecule ligands for targeting QDs to particular proteins on cells. Labeling of malignant cells with QDs can 
be useful for tracking metastases to specific organs and tissues [102]. QDs have shown significant improvement in existing methods for transporting SiRNA to achieve desirable gene silencing effecting a gene-silencing tool [109].

Limitations are associated with visible spectral imaging like autofluorescence (e.g., intestinal tissues) and unsatisfactory tissue penetration by configuration of QDs fluorescing near infrared (NIR) spectra (700-1000nm) [95,110-113]. QDs offer major advantages over radioactive tags or fluorospheres like fluorescin or cyanine dyes in terms of longevity due to their stability and resistance to photobleaching [95]. The intensity of their fluorescent signals as well as capability for multi-conjugation may enhance the level of sensitivity and selectivity. Feasibility of use of QDs for in vivo as well as intraoperative tumor imaging holds promise. Feasibility of use of bioconjugated prostate membrane antigen-targeted QDs to simultaneously image and target animal models have been reported [97].

The inclusion of heavy metals with their composition and potential cytotoxicity may limit the utilization of QDs in human applications [114]. The discharge of toxic metals subsequent to ultraviolet radiation may be limited by modifications of QD like PEGylation and micelle encapsulation $[95,115,116]$.

\section{Carbon Nanotubes}

Carbon nanotubes (CNTs) are allotropes of carbon and have a cylindrical framework. CNTs are formed by rolling graphine sheets into a cylindrical form that can be open ended or capped. They have a high aspect ratio with lengths extending to several micrometers with small diameters of the magnitude of $1 \mathrm{~nm}$. Single-walled nanotubes (SWNT) are CNTs made from a single graphine sheet, and multiwalled carbon nanotubes (MWNTs) are made up with several graphine shets [117]. CNTs are a promising novel carrier system for both small and large therapeutic molecules including drugs, genes and proteins. CNTs can be designed to hold specific functional molecular complexes to their surface to modify their physical or biological properties.

Their unique needle-like shape enables them to be functionalized so that certain molecules could be attached to their surfaces in covalent or noncovalent bonds. Though the mechanism of cell penetration is not fully understood, it is suggested that their needle-like shape enables them to penetrate cellular membranes and enter into intracellular content without significant damage to the cell [118-123]. Significant research on CNTs is centered on their ability of dispensing anticancer agents. Overexpression of folic acid in cancer cells is well known and has led to the design of several nanoparticles with surface modifications to which folic acid derivatives may attach. Further nonspherical nanoparticles (like CNTs) are reported to retain in lymph nodes for prolonged periods of time [40]. Use of CNTs for targeting lymph node cancer has been investigated [124-126].

\section{Gold Nanoparticles, Gold Nanocages, Gold Nanoshells, and Gold Nanorods}

Gold nanoparticles exhibit physicochemical properties that include surface plasmon resonance (SPR), ability to bind amine and thiol groups that enable desired surface engineering, and utilization in medical applications [127]. Gold nanoparticles with their intriguing optical properties can be explored for both imaging and therapeutic applications [128]. Though the earliest gold nanoparticles were gold nanospheres, other types have been developed varying in shape, size, and physical properties, including nanorods, nanoshells, nanocages and surface-enhanced Raman spectroscopy SERS nanoparticles (with excellent SERS properties) to obtain optical properties suitable for biomedical applications. Applications of Gold nanoparticles have been studied in various areas, including in vitro assays, in vitro and in vivo imaging, cancer therapy, and drug delivery. Studies have indicated the use of gold nanoparticles in anticancer drug therapy and radiation therapy [48]. GNPs have been used to enhance drug delivery of oxaliplatin and sensitizers for radiation therapy [48]. GNPs-cetiximabgemcitabine nanocomplex was observed to be more effective both in vitro and in vivo in controlling tumor growth in pancreatic cancer than other agents when delivered by itself or in combination [129]. Using citrate-coated gold nanoparticles of specified size $(2-100 \mathrm{~nm})$ conjugated with a number of trastuzumab antibodies that enable targeting and cross linking of human epidermal growth factor receptor (HER)-2 in human SK-BR-3 breast cancer cells has been shown so that the gold nanoparticles may not only act as drug carriers, but also affect drug-cell interactions and increase the therapeutic effect [130]. Targeted gold nanoparticles, gold nanorods, and gold nanoshells can be used to kill cancer cells using irradiation with a laser of suitable wavelength [48]. Light absorption by gold nanoparticles was suggested to achieve a temperature of $70-80^{\circ} \mathrm{C}$, indicating its use in photothermal therapy [48].

\section{Iron oxide Magnetic NPs}

The capability of surface-antibody conjugation and target specificity under external magnetic control makes magnetic nanoparticles an attractive means for increasing the efficacy for delivering drugs. Superparamagnetic iron oxide nanoparticles (SPIONs) (with $10 \mathrm{~nm}$ mean diameter and superior magnetic properties) are thought to be the most attractive candidates [131,132]. Components of a SPION include magnetite $\left(\mathrm{Fe}_{3} \mathrm{O}_{4}\right)$ or maghemite $\left(\mathrm{Fe}_{2} \mathrm{O}_{3}\right)$, with suitable surface layer to maintain aqueous stability. Methods used for synthesizing SPION include co-precipitation, thermal decomposition, and microemulsion [133]. A number of coating materials including Dextran, albumin, and PEG have been studied for providing biocompatibility to iron oxide nanoparticles [134,135]. A waterdispersible olic acid-pluronic-coated iron oxide magnetic nanoparticle formulation may serve as a universal drug carrier system for systemic administration of water-insoluble drugs along with the possibility of simultaneous imaging and magnetic targeting [136]. In addition to their size, shape, and magnetism crystallinity the diverse qualities of SPIONs include flexibility in fabricating multifunctional SPIONs with fluorescence, and conjugational versatility in targeting ligands as well as drugs. This has led to increasing research in the direction of biomedical applications, especially molecular therapeutics [137]. Gold nanoparticles that are utilized for CT contrast can be integrated with SPIONs forming a hybrid referred as gold iron oxide nanoparticles (GION), that may serve as a contrast agent for both MRI and CT [138]. The hybridized GION offers a better alternative to conventional iodine agents. The hybrid gold-SPION also offers greater enhancement in CT contrast and somewhat higher T2 relaxivity in MRI [133]. The prospects of combining SPION with SPECT probes for MRI/PET and MRI/SPECT dual-modality imaging have raised expectations [139-144]. Magnetic nanoparticles have been conjugated with a number of pharmaceutical agents to treat cancer, including paclitaxel, doxorubicin (DOX), and methotrexate (MTX) [145-147]. 


\section{Ceramic nanoparticles}

Ceramic nanoparticles are porous inorganic complexes. Generating novel applications they have attracted attention as pharmaceutical carriers in the past decade. Ceramic nanoparticles tend to be stable. Biocompatible ceramic nanoparticless such as silica, titania, and alumina that exhibit biocompatibility are utilized in the treatment of cancer. That they lack biodegradability remains the main concern [148]. Hydroxyapatite (HA), zirconia $\left(\mathrm{ZrO}_{2}\right)$, silica $\left(\mathrm{SiO}_{2}\right)$, titanium oxide $\left(\mathrm{TiO}_{2}\right)$, and alumina $\left(\mathrm{Al}_{2} \mathrm{O}_{3}\right)$ are nanoscale ceramics that were configured from new synthetic procedures to amend their physicaland chemical properties with the goal of reducing their cytotoxicity in biological systems. The most sought after application of ceramic nanoparticles in medicine is controlled release of pharmaceuticals, despite adverse host reactions that have been observed in a number of tissues including the immune system [149]. In a comparison of several kinds of ceramic nanoparticles, those composed of silica were observed to be most efficient in transporting therapeutic genes to the spleen and triggering an effective immune response to attack malignant target tissue [150]. Ceramic-based nanoparticless have been used for entrapping agents for photodynamic therapy [151].

The dose and dimensions of nanoparticles are important aspects in biomedical applications. In addition, other characteristics that contribute to its efficiency in controlled drug delivery are high stability, high-load capacity, easy incorporation into hydrophobic and hydrophilic systems, and variability in the ways of administration (i.e., oral, inhalation). Furthermore, the possibility of functionalization of a number of organic groups on its surface opens venues for a directed effect [152].

\section{Conclusion}

The application of nanotechnology in cancer treatment has exhibited significant advancements in recent decades. Nanoparticless offer characteristics for designing and exhibiting properties that may not be feasible with conventional pharmaceutical agents. They show promise in both cancer diagnosis and therapy. Besides the nanoparticles that have obtained approval for clinical use, at present, several other nanoparticle systems are under various stages of preclinical and clinical development and investigation. These include various liposomes, polymeric micelles, dendrimers, QDs, gold nanoparticles, and ceramic nanoparticles. Of the nanoparticles, the potential value of theragnostic nanoparticles is significant. These nanoparticles may be able to carry functions useful for diagnosis and treatment of cancer.

\section{References}

1. Nagahara LA, Lee JS, Molnar LK, Panaro NJ, Farrell D, et al. (2010) Strategic workshops on cancer nanotechnology. Cancer Res 70: 4265-4268.

2. Misra R, Acharya S, Sahoo SK (2010) Cancer nanotechnology: application of nanotechnology in cancer therapy. Drug Discov Today 15: 842-850.

3. Parveen S, Sahoo SK (2006) Nanomedicine: clinical applications of polyethylene glycol conjugated proteins and drugs. Clin Pharmacokinet 45: 965-988.

4. Parveen S, Sahoo SK (2008) Polymeric nanoparticles for cancer therapy. J Drug Target 16: 108-123.

5. Wang X, Yang L, Chen ZG, Shin DM (2008) Application of nanotechnology in cancer therapy and imaging. CA Cancer J Clin 58: 97-110.
6. El-Sayed MA (2001) Some interesting properties of metals confined in time and nanometer space of different shapes. Acc Chem Res 34: 257-264.

7. http://www.twst.com/notes/articles/wac609.html

8. McNeil SE (2005) Nanotechnology for the biologist. J Leukoc Biol 78: 585-594.

9. Sandanaraj BS, Gremlich HU, Kneuer R, Dawson J, Wacha S (2010) Fluorescent nanoprobes as a biomarker for increased vascular permeability: implications in diagnosis and treatment of cancer and inflammation. Bioconjug Chem 21: 93-101.

10. Akiyama Y, Mori T, Katayama Y, Niidome T (2009) The effects of PEG grafting level and injection dose on gold nanorod biodistribution in the tumor-bearing mice. J Control Release 139: 81-84.

11. Stern ST, Hall JB, Yu LL, Wood LJ, Paciotti GF, et al. (2010) Translational considerations for cancer nanomedicine. J Control Release 146: 164-174.

12. Colvin VL, Schlamp MC, Alivisatos AP (1994) Light-emitting diodes made from cadmium selenide nanocrystals and a semiconducting polymer. Nature 370: 354-357.

13. Muthu MS, Singh S (2009) Targeted nanomedicines: effective treatment modalities for cancer, AIDS and brain disorders. Nanomedicine (Lond) 4: 105-118.

14. http://nano.cancer.gov/

15. Wang M, Thanou M (2010) Targeting nanoparticles to cancer. Pharmacol Res 62: 90-99.

16. Vasir JK, Labhasetwar V (2007) Biodegradable nanoparticles for cytosolic delivery of therapeutics. Adv Drug Deliv Rev 59: 718-728.

17. Gabizon A, Goren D, Cohen R, Barenholz Y (1998) Development of liposomal anthracyclines: from basics to clinical applications. J Control Release 53: 275-279.

18. Sahoo SK, Labhasetwar V (2003) Nanotech approaches to drug delivery and imaging. Drug Discov Today 8: 1112-1120.

19. Torchilin V (2008) Antibody-modified liposomes for cancer chemotherapy. Expert Opin Drug Deliv 5: 1003-1025.

20. Kozako T, Arima N, Yoshimitsu M, Honda SI, Soeda S (2012) Liposomes and nanotechnology in drug development: focus on oncotargets. Int J Nanomedicine 7: 4943-4951.

21. Tagawa T, Ueda M (2007) Liposome US200770248541. Google Patents.

22. Isozaki M, Yoshino K, Taguchi K, Kondo M (2008) Liposome Preparation US20080279916 A1. Google Patents.

23. Allen TM, Sapra P, Moase E, Moreira J, Iden D (2002) Adventures in targeting. J Liposome Res 12: 5-12.

24. Ferrari M (2005) Cancer nanotechnology: opportunities and challenges. Nat Rev Cancer 5: 161-171.

25. Fassas A, Anagnostopoulos A (2005) The use of liposomal daunorubicin (DaunoXome) in acute myeloid leukemia. Leuk Lymphoma 46: 795-802.

26. Treat J, Greenspan A, Forst D, Sanchez JA, Ferrans VJ, et al. (1990) Antitumor activity of liposome-encapsulated doxorubicin in advanced breast cancer: phase II study. J Natl Cancer Inst 82: 1706-1710.

27. Forssen EA, Tökés ZA (1983) Improved therapeutic benefits of doxorubicin by entrapment in anionic liposomes. Cancer Res 43: 546-550.

28. Straubinger RM, Lopez NG, Debs RJ, Hong K, Papahadjopoulos D (1988) Liposome-based therapy of human ovarian cancer: parameters determining potency of negatively charged and antibody-targeted liposomes. Cancer Res 48: 5237-5245.

29. Robert NJ, Vogel CL, Henderson IC, Sparano JA, Moore MR, et al. (2004) The role of the liposomal anthracyclines and other systemic therapies in the management of advanced breast cancer. Semin Oncol 31: 106-146.

30. Zheng J, Perkins G, Kirilova A, Allen C, Jaffray DA (2006) Multimodal contrast agent for combined computed tomography and magnetic resonance imaging applications. Invest Radiol 41: 339-348. 
31. Jaffray D, Allen C, Zheng J, Reilly RM, Perkins GJ (2008) Compositions and Method for Multimodal Imaging US20080206131 A1. Google Patents.

32. Scheffel U, Rhodes BA, Natarajan TK, Wagner HN Jr (1972) Albumin microspheres for study of the reticuloendothelial system. J Nucl Med 13: 498-503.

33. Müller RH, Mäder K, Gohla S (2000) Solid lipid nanoparticles (SLN) for controlled drug delivery - a review of the state of the art. Eur J Pharm Biopharm 50: 161-177.

34. Cavalli R, Caputo O, Carlotti ME, Trotta M, Scarnecchia C, et al. (1997) Sterilization and freeze-drying of drug-free and drug-loaded solid lipid nanoparticles. Int J Pharm 148: 47-54.

35. Almeida AJ, Souto E (2007) Solid lipid nanoparticles as a drug delivery system for peptides and proteins. Adv Drug Deliv Rev 59: 478-490.

36. Wong HL, Bendayan R, Rauth AM, Li Y, Wu XY (2007) Chemotherapy with anticancer drugs encapsulated in solid lipid nanoparticles. Adv Drug Deliv Rev 59: 491-504.

37. Wissing SA, Kayser O, Müller RH (2004) Solid lipid nanoparticles for parenteral drug delivery. Adv Drug Deliv Rev 56: 1257-1272.

38. Hamaguchi T, Kato K, Yasui H, Morizane C, Ikeda M, et al. (2007) A phase I and pharmacokinetic study of NK105, a paclitaxel-incorporating micellar nanoparticle formulation. Br J Cancer 97: 170-176.

39. Teeranachaideekul V, Müller RH, Junyaprasert VB (2007) Encapsulation of ascorbyl palmitate in nanostructured lipid carriers (NLC)--effects of formulation parameters on physicochemical stability. Int J Pharm 340: 198-206.

40. Radtke ML, Souto EB, Müller RH (2005) Nanostructured lipid carriers: a novel generation of solid lipid drug carriers. Pharmaceut Tech Eur 17: 45-50.

41. Murthy RSR (2005) Solid lipid nanoparticles as carriers for anti-cancer drugs to solid tumours. Drug Deliv 12: 385-392.

42. Ruckmani K, Sivakumar M, Ganeshkumar PA (2006) Methotrexate loaded solid lipid nanoparticles (SLN) for effective treatment of carcinoma. J Nanosci Nanotechnol 6: 2991-2995.

43. Yang SC, Lu LF, Cai Y, Zhu JB, Liang BW, et al. (1999) Body distribution in mice of intravenously injected camptothecin solid lipid nanoparticles and targeting effect on brain. J Control Release 59: 299-307.

44. Lu B, Xiong SB, Yang H, Yin XD, Chao RB (2006) Solid lipid nanoparticles of mitoxantrone for local injection against breast cancer and its lymph node metastases. Eur J Pharm Sci 28: 86-95.

45. Wong HL, Rauth AM, Bendayan R, Manias JL, Ramaswamy M, et al. (2006) A new polymer-lipid hybrid nanoparticle system increases cytotoxicity of doxorubicin against multidrug-resistant human breast cancer cells. Pharm Res 23: 1574-1585.

46. Zhang XG, Miao J, Dai YQ, Du YZ, Yuan H, et al. (2008) Reversal activity of nanostructured lipid carriers loading cytotoxic drug in multidrug resistant cancer cells. Int J Pharm 361: 239-244.

47. Bondi ML, Craparo EF, Giammona G, Cervello M, Azzolina A, et al (2007) Nanostructured lipid carriers-containing anticancer compounds: preparation, characterization, and cytotoxicity studies. Drug Deliv 14: 61-67.

48. Chithrani DB (2010) Nanoparticles for improved therapeutics and imaging in cancer therapy. Recent Pat Nanotechnol 4: 171-180.

49. Soppimath KS1, Aminabhavi TM, Kulkarni AR, Rudzinski WE (2001) Biodegradable polymeric nanoparticles as drug delivery devices. J Control Release 70: 1-20.

50. Hans ML, Lowman AM (2002) Biodegradable nanoparticles for drug delivery and targeting. Curr Opinion Solid State Mater Sci 6: 319-327.

51. Arias JL, Gallardo V, Gómez-Lopera SA, Plaza RC, Delgado AV (2001) Synthesis and characterization of poly(ethyl-2-cyanoacrylate) nanoparticles with a magnetic core. J Control Release 77: 309-321.

52. Fontana G, Pitarresi G, Tomarchio V, Carlisi B, San Biagio PL (1998) Preparation, characterization and in vitro antimicrobial activity of ampicillin-loaded polyethylcyanoacrylate nanoparticles. Biomaterials 19: 1009-1017.
53. Kim SY, Lee YM (2001) Taxol-loaded block copolymer nanospheres composed of methoxy poly(ethylene glycol) and poly(epsiloncaprolactone) as novel anticancer drug carriers. Biomaterials 22: 1697-1704.

54. Panyam J, Labhasetwar V (2003) Biodegradable nanoparticles for drug and gene delivery to cells and tissue. Adv Drug Deliv Rev 55: 329-347.

55. http://www.nanopharmaceuticals.org/Polymeric_nanoparticles.html

56. Kumari A1, Yadav SK, Yadav SC (2010) Biodegradable polymeric nanoparticles based drug delivery systems. Colloids Surf B Biointerfaces 75: 1-18.

57. Hawker CJ, Frechet JMJ (1990) Preparation of polymers with controlled molecular architecture. A new convergent approach to dendritic macromolecules. J Am Chem Soc 112: 7638-7647.

58. Svenson S, Tomalia DA (2005) Dendrimers in biomedical applicationsreflections on the field. Adv Drug Deliv Rev 57: 2106-2129.

59. Oerlemans C, Bult W, Bos M, Storm G, Nijsen JF, et al. (2010) Polymeric micelles in anticancer therapy: targeting, imaging and triggered release. Pharm Res 27: 2569-2589.

60. Rawat M, Singh D, Saraf S, Saraf S (2006) Nanocarriers: promising vehicle for bioactive drugs. Biol Pharm Bull 29: 1790-1798.

61. Adams ML, Lavasanifar A, Kwon GS (2003) Amphiphilic block copolymers for drug delivery. J Pharm Sci 92: 1343-1355.

62. Xiong MP, Kwon GS, Bae Y, Kataoka K (2009) Compositions for delivery of therapeutic agents US20090232762 A1. Google Patents.

63. Torchilin VP, Lukyanov AN, Gao Z, Papahadjopoulos-Sternberg B (2003) Immunomicelles: targeted pharmaceutical carriers for poorly soluble drugs. Proc Natl Acad Sci U S A 100: 6039-6044.

64. Kataoka K, Matsumoto T, Yokoyama M, Okano T, Sakurai Y, et al. (2000) Doxorubicin-loaded poly(ethylene glycol)-poly(beta-benzyl-Laspartate) copolymer micelles: their pharmaceutical characteristics and biological significance. J Control Release 64: 143-153.

65. Chatterjee DK, Fong LS, Zhang Y (2008) Nanoparticles in photodynamic therapy: an emerging paradigm. Adv Drug Deliv Rev 60: 1627-1637.

66. Wolinsky JB, Grinstaff MW (2008) Therapeutic and diagnostic applications of dendrimers for cancer treatment. Adv Drug Deliv Rev 60: 1037-1055.

67. Lee H, Hoang B, Fonge H, Reilly RM, Allen C (2010) In vivo distribution of polymeric nanoparticles at the whole-body, tumor, and cellular levels. Pharm Res 27: 2343-2355.

68. Hoang B, Lee H, Reilly RM, Allen C (2009) Noninvasive monitoring of the fate of 111In-labeled block copolymer micelles by high resolution and high sensitivity microSPECT/CT imaging. Mol Pharm 6: 581-592.

69. Shiraishi K, Kawano K, Minowa T, Maitani Y, Yokoyama M (2009) Preparation and in vivo imaging of PEG-poly(L-lysine)-based polymeric micelle MRI contrast agents. J Control Release 136: 14-20.

70. Talelli M, Rijcken CJ, Lammers T, Seevinck PR, Storm G, et al. (2009) Superparamagnetic iron oxide nanoparticles encapsulated in biodegradable thermosensitive polymeric micelles: toward a targeted nanomedicine suitable for image-guided drug delivery. Langmuir 25 2060-2067.

71. Khemtong C, Kessinger CW, Togao O, Ren J, Takahashi M, et al. (2009) Off-resonance saturation magnetic resonance imaging of superparamagnetic polymeric micelles. Conf Proc IEEE Eng Med Biol Soc 2009: 4095-4097.

72. Lu J, Ma S, Sun J, Xia C, Liu C, et al. (2009) Manganese ferrite nanoparticle micellar nanocomposites as MRI contrast agent for liver imaging. Biomaterials 30: 2919-2928.

73. Nasongkla N, Bey E, Ren J, Ai H, Khemtong C, et al. (2006) Multifunctional polymeric micelles as cancer-targeted, MRIultrasensitive drug delivery systems. Nano Lett 6: 2427-2430.

74. Ai H, Duerk J, Flask C, Gao J, Lewin J, et al. (2007) Dual Function Polymer Micelles US20070253899. Google Patents.

75. Tomalia DA, Baker H, Dewald J, Hall M, Kallos G, et al. (1985) A New Class of Polymers: Starburst-Dendritic Macromolecules. Polymer J 17: $117-132$. 
76. Barrett T, Ravizzini G, Choyke PL, Kobayashi H (2009) Dendrimers in medical nanotechnology. IEEE Eng Med Biol Mag 28: 12-22.

77. Antoni P, Hed Y, Nordberg A, Nyström D, von Holst H, et al. (2009) Bifunctional dendrimers: from robust synthesis and accelerated one-pot postfunctionalization strategy to potential applications. Angew Chem Int Ed Engl 48: 2126-2130.

78. McElhanon JR, McGrath DV (2000) Toward chiral polyhydroxylated dendrimers. Preparation and chiroptical properties J Org Chem 65: 3525-3529.

79. Liang CO, Fréchet JMJ (2005) Incorporation of Functional Guest Molecules into an Internally Functionalizable Dendrimer through Olefin Metathesis. Macromolecules 38: 6276-6284.

80. Menjoge AR, Kannan RM, Tomalia DA (2010) Dendrimer-based drug and imaging conjugates: design considerations for nanomedical applications. Drug Discov Today 15: 171-185.

81. Tekade RK, Kumar PV, Jain NK (2009) Dendrimers in oncology: an expanding horizon. Chem Rev 109: 49-87.

82. D'Emanuele A, Attwood D (2005) Dendrimer-drug interactions. Adv Drug Deliv Rev 57: 2147-2162.

83. Jansen JF, de Brabander-van den Berg EM, Meijer EW (1994) Encapsulation of guest molecules into a dendritic box. Science 266: 1226-1229.

84. Wiener EC, Brechbiel MW, Brothers H, Magin RL, Gansow OA, et al. (1994) Dendrimer-based metal chelates: a new class of magnetic resonance imaging contrast agents. Magn Reson Med 31: 1-8.

85. Wiener EC, Konda S, Shadron A, Brechbiel M, Gansow O (1997) Targeting dendrimer-chelates to tumors and tumor cells expressing the high-affinity folate receptor. Invest Radiol 32: 748-754

86. Quintana A, Raczka E, Piehler L, Lee I, Myc A, et al. (2002) Design and function of a dendrimer-based therapeutic nanodevice targeted to tumor cells through the folate receptor. Pharm Res 19: 1310-1316.

87. Ooya T, Lee J, Park K (2004) Hydrotropic dendrimers of generations 4 and 5: synthesis, characterization, and hydrotropic solubilization of paclitaxel. Bioconjug Chem 15: 1221-1229.

88. Neerman MF, Chen HT, Parrish AR, Simanek EE (2004) Reduction of drug toxicity using dendrimers based on melamine. Mol Pharm 1: 390-393.

89. Yang W, Barth RF, Adams DM, Soloway AH (1997) Intratumoral delivery of boronated epidermal growth factor for neutron capture therapy of brain tumors. Cancer Res 57: 4333-4339.

90. Yang W, Barth RF, Adams DM, Ciesielski MJ, Fenstermaker RA, et al (2002) Convection-enhanced delivery of boronated epidermal growth factor for molecular targeting of EGF receptor-positive gliomas. Cancer Res 62: 6552-6558.

91. Kobayashi H, Kawamoto S, Saga T, Sato N, Ishimori T, et al. (2001) Avidin-dendrimer-(1B4M-Gd)(254): a tumor-targeting therapeutic agent for gadolinium neutron capture therapy of intraperitoneal disseminated tumor which can be monitored by MRI. Bioconjug Chem 12: 587-593.

92. Shi X, Wang S, Meshinchi S, Van Antwerp ME, Bi X, et al. (2007) Dendrimer-entrapped gold nanoparticles as a platform for cancer-cell targeting and imaging. Small 3: 1245-1252.

93. Fu Y, Nitecki DE, Maltby D, Simon GH, Berejnoi K, et al. (2006) Dendritic iodinated contrast agents with PEG-cores for CT imaging: synthesis and preliminary characterization. Bioconjug Chem 17: 1043-1056.

94. Gunasekera UA, Pankhurst QA, Douek M (2009) Imaging applications of nanotechnology in cancer. Target Oncol 4: 169-181.

95. Cuenca AG, Jiang H, Hochwald SN, Delano M, Cance WG, et al. (2006) Emerging implications of nanotechnology on cancer diagnostics and therapeutics. Cancer 107: 459-466.

96. Alper J (2005) Shining a Light on Cancer Research. NCI Alliance for Nanotechnology in Cancer January/February: 1-3.

97. http://www.americanelements.com/nanotech.htm
98. Bruchez M Jr, Moronne M, Gin P, Weiss S, Alivisatos AP (1998) Semiconductor nanocrystals as fluorescent biological labels. Science 281: 2013-2016.

99. Seydel C (2003) Quantum dots get wet. Science 300: 80-81.

100. Michalet X, Pinaud FF, Bentolila LA, Tsay JM, Doose S, et al. (2005) Quantum dots for live cells, in vivo imaging, and diagnostics. Science 307: 538-544.

101. Qu L, Peng X (2002) Control of photoluminescence properties of CdSe nanocrystals in growth. J Am Chem Soc 124: 2049-2055.

102. Voura EB, Jaiswal JK, Mattoussi H, Simon SM (2004) Tracking metastatic tumor cell extravasation with quantum dot nanocrystals and fluorescence emission-scanning microscopy. Nat Med 10: 993-998.

103. Gao X, Cui Y, Levenson RM, Chung LW, Nie S (2004) In vivo cancer targeting and imaging with semiconductor quantum dots. Nat Biotechnol 22: 969-976.

104. http://newscenter.lbl.gov/feature-stories/2009/01/05/better-way-tomake-nanotubes/

105. Dahan M, Lévi S, Luccardini C, Rostaing P, Riveau B, et al. (2003) Diffusion dynamics of glycine receptors revealed by single-quantum dot tracking. Science 302: 442-445.

106. Howarth M, Liu W, Puthenveetil S, Zheng Y, Marshall LF, et al. (2008) Monovalent, reduced-size quantum dots for imaging receptors on living cells. Nat Methods 5: 397-399.

107. Akerman ME, Chan WC, Laakkonen P, Bhatia SN, Ruoslahti E (2002) Nanocrystal targeting in vivo. Proc Natl Acad Sci U S A 99: 12617-12621.

108. Farlow J, Seo D, Broaders KE, Taylor MJ, Gartner ZJ, et al. (2013) Formation of targeted monovalent quantum dots by steric exclusion. Nat Methods 10: 1203-1205.

109. Tan WB, Jiang S, Zhang Y (2007) Quantum-dot based nanoparticles for targeted silencing of HER2/neu gene via RNA interference. Biomaterials 28: $1565-1571$.

110. Frangioni JV (2003) In vivo near-infrared fluorescence imaging. Curr Opin Chem Biol 7: 626-634.

111. Parungo CP, Ohnishi S, De Grand AM, Laurence RG, Soltesz EG, et al. (2004) In vivo optical imaging of pleural space drainage to lymph nodes of prognostic significance. Ann Surg Oncol 11: 1085-1092.

112. Parungo CP, Colson YL, Kim SW, Kim S, Cohn LH, et al. (2005) Sentinel lymph node mapping of the pleural space. Chest 127: 1799-1804.

113. Parungo CP, Ohnishi S, Kim SW, Kim S, Laurence RG, et al. (2005) Intraoperative identification of esophageal sentinel lymph nodes with near-infrared fluorescence imaging. J Thorac Cardiovasc Surg 129: 844-850.

114. Derfus AM, Chan WCW, Bhatia SN (2004) Probing the Cytotoxicity of Semiconductor Quantum Dots. Nano Lett 4: 11-18.

115. Gao X, Yang L, Petros JA, Marshall FF, Simons JW, et al. (2005) In vivo molecular and cellular imaging with quantum dots. Curr Opin Biotechnol 16: 63-72.

116. Stroh M, Zimmer JP, Duda DG, Levchenko TS, Cohen KS, et al. (2005) Quantum dots spectrally distinguish multiple species within the tumor milieu in vivo. Nat Med 11: 678-682.

117. Elhissi AM, Ahmed W, Hassan IU, Dhanak VR, D'Emanuele A (2012) Carbon nanotubes in cancer therapy and drug delivery. J Drug Deliv 2012: 837327.

118. Pantarotto D, Briand JP, Prato M, Bianco A (2004) Translocation of bioactive peptides across cell membranes by carbon nanotubes. Chem Commun (Camb) : 16-17.

119. Pantarotto D, Singh R, McCarthy D, Erhardt M, Briand JP, et al. (2004) Functionalized carbon nanotubes for plasmid DNA gene delivery. Angew Chem Int Ed Engl 43: 5242-5246.

120. Kam NW, Liu Z, Dai H (2006) Carbon nanotubes as intracellular transporters for proteins and DNA: an investigation of the uptake mechanism and pathway. Angew Chem Int Ed Engl 45: 577-581.

121. Kam NW, Liu Z, Dai H (2005) Functionalization of carbon nanotubes via cleavable disulfide bonds for efficient intracellular delivery of siRNA and potent gene silencing. J Am Chem Soc 127: 12492-12493. 
122. Klumpp C, Kostarelos K, Prato M, Bianco A (2006) Functionalized carbon nanotubes as emerging nanovectors for the delivery of therapeutics. Biochim Biophys Acta 1758: 404-412.

123. Cai D, Mataraza JM, Qin ZH, Huang Z, Huang J, et al. (2005) Highly efficient molecular delivery into mammalian cells using carbon nanotube spearing. Nat Methods 2: 449-454.

124. Yang F, Fu de L, Long J, Ni QX (2008) Magnetic lymphatic targeting drug delivery system using carbon nanotubes. Med Hypotheses 70: 765-767.

125. Yang F, Hu J, Yang D, Long J, Luo G, et al. (2009) Pilot study of targeting magnetic carbon nanotubes to lymph nodes. Nanomedicine (Lond) 4: 317-330.

126. Yang L, Ng KY, Lillehei KO (2003) Cell-mediated immunotherapy: a new approach to the treatment of malignant glioma. Cancer Control 10: 138-147.

127. Shukla R, Bansal V, Chaudhary M, Basu A, Bhonde RR, et al. (2005) Biocompatibility of gold nanoparticles and their endocytotic fate inside the cellular compartment: a microscopic overview. Langmuir 21: 10644-10654.

128. Cai W, Gao T, Hong H, Sun J (2008) Applications of gold nanoparticles in cancer nanotechnology. Nanotechnol Sci Appl : S3788.

129. Patra CR, Bhattacharya R, Wang E, Katarya A, Lau JS, et al. (2008) Targeted delivery of gemcitabine to pancreatic adenocarcinoma using cetuximab as a targeting agent. Cancer Res 68: 1970-1978.

130. Jiang W, Kim BY, Rutka JT, Chan WC (2008) Nanoparticle-mediated cellular response is size-dependent. Nat Nanotechnol 3: 145-150.

131. Laurent S, Mahmoudi M (2011) Superparamagnetic iron oxide nanoparticles: promises for diagnosis and treatment of cancer. Int J Mol Epidemiol Genet 2: 367-390.

132. Mahmoudi M, Stroeve P, Milani AS, Arbab AS (2011) Superparamagnetic Iron Oxide Nanoparticles: Synthesis, Surface Engineering, Cytotoxicity and Biomedical Applications (Nanotechnology Science and Technology). Nova Science Publishers, Inc., Hauppauge, NY.

133. Thomas R, Park IK, Jeong YY (2013) Magnetic iron oxide nanoparticles for multimodal imaging and therapy of cancer. Int J Mol Sci 14: 15910-15930.

134. Berry CC, Wells S, Charles S, Curtis AS (2003) Dextran and albumin derivatised iron oxide nanoparticles: influence on fibroblasts in vitro. Biomaterials 24: 4551-4557.

135. Gupta AK, Curtis AS (2004) Surface modified superparamagnetic nanoparticles for drug delivery: interaction studies with human fibroblasts in culture. J Mater Sci Mater Med 15: 493-496.

136. Jain TK, Morales MA, Sahoo SK, Leslie-Pelecky DL, Labhasetwar V (2005) Iron oxide nanoparticles for sustained delivery of anticancer agents. Mol Pharm 2: 194-205.

137. Jun YW, Seo JW, Cheon J (2008) Nanoscaling laws of magnetic nanoparticles and their applicabilities in biomedical sciences. Acc Chem Res 41: 179-189.

138. Kim D, Kim JW, Jeong YY, Jon S (2009) Antibiofouling Polymer Coated Gold@Iron Oxide Nanoparticle (GION) as a Dual Contrast Agent for CT and MRI. Bull Korean Chem 30: 1855-1857.
139. Yang X, Hong H, Grailer JJ, Rowland IJ, Javadi A, et al. (2011) cRGDfunctionalized, DOX-conjugated, and ${ }^{64} \mathrm{Cu}$-labeled superparamagnetic iron oxide nanoparticles for targeted anticancer drug delivery and PET/MR imaging. Biomaterials 32: 4151-4160.

140. Lee HY, Li Z, Chen K, Hsu AR, Xu C, et al. (2008) PET/MRI dualmodality tumor imaging using arginine-glycine-aspartic (RGD)conjugated radiolabeled iron oxide nanoparticles. J Nucl Med 49: 1371-1379.

141. Torres Martin de Rosales R1, Tavaré R, Paul RL, Jauregui-Osoro M, Protti A, et al. (2011) Synthesis of $64 \mathrm{Cu}(\mathrm{II})$ bis(dithiocarbamatebisphosphonate) and its conjugation with superparamagnetic iron oxide nanoparticles: in vivo evaluation as dualmodality PET-MRI agent. Angew Chem Int Ed Engl 50: 5509-5513.

142. Madru R, Kjellman P, Olsson F, Wingårdh K, Ingvar C, et al. (2012) $99 \mathrm{mTc}$-labeled superparamagnetic iron oxide nanoparticles for multimodality SPECT/MRI of sentinel lymph nodes. J Nucl Med 53: 459-463.

143. Misri R, Meier D, Yung AC, Kozlowski P, Häfeli UO (2012) Development and evaluation of a dual-modality (MRI/SPECT) molecular imaging bioprobe. Nanomedicine 8: 1007-1016.

144. Torres Martin de Rosales R, Tavaré R, Glaria A, Varma G, Protti A, et al. (2011) ( $\left.\hat{a}^{1} \hat{a}^{1} \mathrm{~m}\right)$ Tc-bisphosphonate-iron oxide nanoparticle conjugates for dual-modality biomedical imaging. Bioconjug Chem 22: 455-465.

145. Park JH, von Maltzahn G, Ruoslahti E, Bhatia SN, Sailor MJ (2008) Micellar hybrid nanoparticles for simultaneous magnetofluorescent imaging and drug delivery. Angew Chem Int Ed Engl 47: 7284-7288.

146. Santra S, Kaittanis C, Grimm J, Perez JM (2009) Drug/dye-loaded, multifunctional iron oxide nanoparticles for combined targeted cancer therapy and dual optical/magnetic resonance imaging. Small 5: 1862-1868.

147. Quan Q, Xie J, Gao H, Yang M, Zhang F, et al. (2011) HSA coated iron oxide nanoparticles as drug delivery vehicles for cancer therapy. Mol Pharm 8: 1669-1676.

148. Cherian AK, Rana AC, Jain SK (2000) Self-assembled carbohydratestabilized ceramic nanoparticles for the parenteral delivery of insulin. Drug Dev Ind Pharm 26: 459-463.

149. Moreno-Vega AI, Gómez-Quintero T, Nuñez-Anita RE, Acosta-Torres LS, Castaño V (2012) Polymeric and Ceramic Nanoparticles in Biomedical Applications. J Nanotechnol: 1-10.

150. Tan K, Cheang P, Ho IA, Lam PY, Hui KM (2007) Nanosized bioceramic particles could function as efficient gene delivery vehicles with target specificity for the spleen. Gene Ther 14: 828-835.

151. Roy I, Ohulchanskyy TY, Pudavar HE, Bergey EJ, Oseroff AR, et al. (2003) Ceramic-based nanoparticles entrapping water-insoluble photosensitizing anticancer drugs: a novel drug-carrier system for photodynamic therapy. J Am Chem Soc 125: 7860-7865.Fadeel B, GarciaBennett AE (2010) Better safe than sorry: Understanding the toxicological properties of inorganic nanoparticles manufactured for biomedical applications. Adv Drug Deliv Rev 62: 362-374.
This article was originally published in a special issue, entitled: "Cancer Radiation Therapy", Edited by University of Arkansas for Medical Sciences, USA 